\title{
Geometric probability based stereological corrections
}

\author{
S. L. Gay* $\quad$ J. M. Keith ${ }^{\dagger}$ \\ (Received 7 August 2003; revised 15 July 2004)
}

\begin{abstract}
In mineral processing, a classic problem is that of estimating the particle composition distribution from particle sections. This problem occurs because mineralogical analysis is performed on particle sections rather than the particles. Thus one wishes to infer three-dimensional information (particle composition distribution) from two-dimensional information (particle sections). There are a number of different techniques available for estimating the particle composition distribution and these are called stereological corrections. This paper provides examples of very simple stereological corrections based on geometric probability equations as well as the principle of maximum entropy. The paper focuses on three methods. The first method is a simple
\end{abstract}

*Julius Kruttschnitt Mineral Research Centre, The University of Queensland, Isles Rd., Indooroopilly 4068 Australia. mailto: s.gay@uq.edu.au

${ }^{\dagger}$ Department of Mathematics, The University of Queensland, 4066, Australia. mailto:keith1@mailbox.uq.edu.au

See http://anziamj.austms.org.au/V45/CTAC2003/Gays/home.html for this article, (c) Austral. Mathematical Soc. 2004. Published July 17, 2004. ISSN 1446-8735 
entropy based model based on particle section information only. The second method also uses entropy, but the entropy is based on relating particles to particle sections, and is therefore more complex than the first. The third model uses both the particle section distribution and the linear intercept distribution. The most accurate model is that which uses both particle sections and linear intercepts.

\section{Contents}

1 Introduction

C619

2 Geometric probability

C621

3 Methods of solution

C624

3.1 A simple entropy method using particle sections . . . . . . C624

3.2 Method using relationships between particles and particle sec-

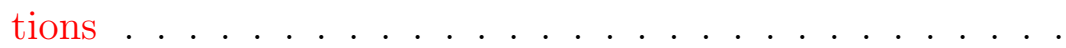

C626

3.3 Simple model using both linear intercepts and planar sections C627

4 Discussion

C628

5 Conclusions

C630

References

C630

\section{Introduction}

In order to optimize a mineral processing plant, an important consideration is the particle composition distribution. In particular, one would like to know the proportion of particles that are 'liberated' (containing only valuable mineral), 'composite' (containing more than one mineral) and 'barren' 


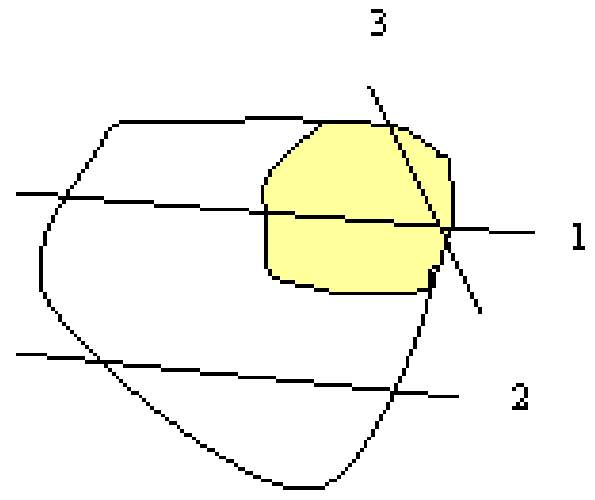

FIGURE 1: Schematic diagram with three linear intercepts through a two dimensional particle. Even though the particle is composite, the intercepts can still appear liberated (intercepts $2 \& 3$ ). This problem is analogous to intersecting particles with planar intercepts

(containing no valuable mineral). For mineral processing operations, one wishes to maximize production and concentration of liberated particles.

The method of preparing particles for mineralogical analysis is to randomly place them in a mount. The complete mount is then sectioned (normally only once) revealing a representative sample of particle sections. Because of the problem shown in Figure 1, when a set of particles are subject to mineralogical analysis, the proportion of particle sections that appears liberated is generally more than the actual proportion of liberated particles. Assuming that particles are indeed mounted randomly and isotropically, the resultant particle sections are more fully described as isotropic, uniform, random particle sections.

The general term given to estimating particle composition distribution using particle section information is called a stereological correction. The stereological correction problem for mineral processing was first recognised 
by Gaudin [4]. He developed a simple stereological correction procedure, where particles were assumed to be spherical caps, and those particles that were composite were assumed to behave under sectioning as if they had a composition of $50 \%$.

The spherical cap shape, assumed by Gaudin, was fairly limited as minerals could actually be finely dispersed throughout particles. In this case the stereological correction would be small, and much less than that assumed by Gaudin. Thus Gaudin's method became a methodology for estimating the maximum correction required.

Gaudin's approach lead naturally to the models developed by Jones and Horton [10], and Hill et al. [7] who removed the assumption that composite particles behaved as if they had a composition of $50 \%$, but allowed a full distribution of particles and particle sections. In order to successfully apply these models it was necessary to determine the complete distribution of particle sections arising from particles of particular compositions. This transformation method is called the kernel.

There have been many other methods developed to solve the problem. The main methods are the kernel estimation methods of Miller and Lin [13], King and Schneider [12]; and the geometric probability approaches of Barbery [1], Gay [5] and Keith [11]. The geometric probability approach is largely based on the equation developed by Davy [2].

\section{Geometric probability}

For the purposes of this paper, stereology is used as a general term that means the relationship between particles and particle sections, whereas geometric probability is used to represent those relationships that are independent of particle shape and grain structure. The most basic geometric probability is 


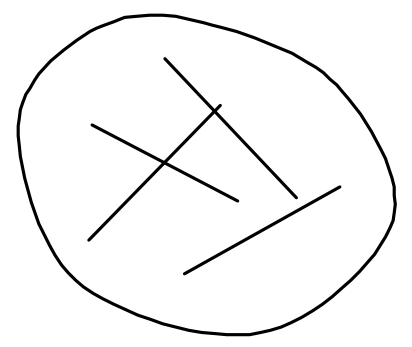

Figure 2: Schematic diagram showing how the average distance between pixel points is estimated. Pairs of points are chosen randomly and the distance between pairs is calculated. The average of the distance values is $d$

that of Delesse [3]:

$$
\frac{E_{v}\left(c_{v} V\right)}{E_{v}(V)}=\frac{E_{a}\left(c_{a} A\right)}{E_{a}(A)} .
$$

Here $c_{v}$ is the volume composition of each particle, $V$ is the volume of each particle, $c_{a}$ is the area composition of each particle section, $A$ is the area of each particle section, $E_{v}$ is the average with respect to particles and $E_{a}$ is the average with respect to particle sections. Here the particle information is unknown, whereas the particle section information is known. The particle referred to in the average refers to all particles in the mount, and not specifically to those that are sectioned.Delesse's equation implies that the average composition of particles equals the average composition of particle sections. However, the equation does not provide any insights into the problem of estimating how many particles are liberated.

Davy [2] described an equation to estimate the variance of the particle composition distribution. Davy derived this equation in a manner that im- 


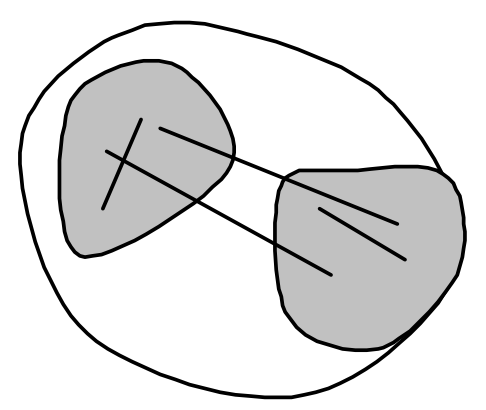

FiguRE 3: Schematic diagram showing how the average distance between pixel points is estimated. Pairs of points are chosen randomly and the distance between pairs is calculated. The average of the distance values is $d_{11}$

plied that one must know the texture of the ore before breakage. Gay [6] presented a modified and much simplified version of the equation that is more appropriate to use on particle section data. The equation is based on point sampling pixel data, and requires estimation of average distances between pixels on particle sections. In particular two key parameters are: $d_{p p}$ (which is the average distance between particle pixels on the same planar section); and $d_{11}$ (which is the average distance between mineral 1 pixels on the same planar section). Estimation of these parameters is shown in Figures 2 and 3. Here Gay's equation is presented for binary particles (consisting of at most two minerals) and the two minerals are identified as mineral 1 (the mineral of interest) and mineral 2 (the associated gangue). Gay's equation is

$$
\frac{E_{v}\left(c_{v}^{2} V^{2}\right)}{E_{v}\left(V^{2}\right)}=\frac{E_{a}\left(c_{a}^{2} d_{11} A^{2}\right)}{E_{a}\left(d_{p p} A^{2}\right)} .
$$

If particle size is independent of particle composition, then, for the left-hand 
side of equation (1), the volume terms can be removed.

Thus the left-hand terms in equations (1-2)

\section{Methods of solution}

The stereological problem does not have a set solution; and there are many possible methods. In this paper, focus is only given to methods based on geometric probability and the principle of maximum entropy. The various methods of solution are grouped into:

- a simple entropy method using particle sections;

- an entropy method based on particle sections only, but more detailed than the simple entropy method;

- a simple method based on both linear intercepts and particle sections

These methods are discussed in the following sections.

\subsection{A simple entropy method using particle sections}

If grain size is infinitesimally small, then it follows that:

- the parameters $d_{11}$ and $d_{p p}$ given in Section 2 will be the same value;

- the composition of each particle sections will be the same as for that of the particle from which they were derived; and

- the second moment of the particle composition distribution equals that of the second moment of particle composition distribution. 
The latter property is also true conversely. That is, if the second moments of the particle composition distribution and the particle section composition distribution are the same, then these two distributions must be the same. This information can be used in the estimation procedure; however, there is still insufficient information available to determine the complete distribution. A mathematical approach for estimating distributions where there is insufficient information is the entropy approach. This method is used to formulate a 'likely' distribution given the available information. Informative references are by Jaynes [8, 9] and Shannon and Weaver [14].

In order to formulate the problem, the composition distribution is divided into composition classes (also called bins) with the twelve bins being composition classes [0\%], (0-10\%], (10-20\%], .., [100\%]. $l_{v, i}$ denotes the particle composition distribution (with $i$ representing particle class), and $l_{a, i}$ is the particle section composition distribution. Thus the probability entropy is formulated as

$$
H=-\sum_{i=1} l_{v, i}\left[\ln \left(l_{v, i}\right)-\ln \left(l_{a, i}\right)\right],
$$

constrained by, from (1),

$$
\sum_{i} l_{v, i} c_{i}=\overline{c_{v}}
$$

and, from (2),

$$
\sum_{i} l_{v, i} c_{i}^{2}=\overline{c_{v}^{2}}
$$

There is also the natural constraint:

$$
\sum_{i} l_{v, i}=1
$$

The problem then is to maximise the probability entropy, constrained by equations (4-6). The solution is easily determined using Lagrange multipliers. Assign: $\lambda$ to equation (4), $\mu$ to equation (5), and $a$ to equation (6). The solution is

$$
\ln \left(l_{a, i}\right)-\ln \left(l_{v, i}\right)-1+\lambda c_{i}+\mu c_{i}^{2}+a=0 .
$$


With the substitution

$$
A=e^{a-1},
$$

the solution becomes

$$
l_{v, i}=A l_{a, i} \exp \left(\lambda c_{i}+\mu c_{i}^{2}\right) .
$$

The parameters $A, \lambda$ and $\mu$ are determined so as to satisfy the constraints.

\subsection{Method using relationships between particles and particle sections}

Keith [11] developed a model based on entropy and geometric probability, which involves determining the probability relationship between particles and particle sections. For example, what is the probability that a particle section of a particular composition originally came from a particle of a particular composition. Here a simplified version of Keith's model is represented. Because this method uses more equations than the simple entropy model it is expected to perform better.

Consider the composition distributions (particles and particle sections) split into the 12 conventional bins. Denote $p_{i, j}$ as the probability that a particle section in the $j$ th particle section composition bin came from a particle in the $i$ th particle composition bin. The particle composition distribution is then

$$
l_{v, i}=\sum_{j} l_{a, j} p_{i, j} .
$$

With this probability representation, Delesse's equation (1) applied to each particle bin (represented by $i$ ) gives

$$
\sum_{j} l_{a, j} p_{i, j}\left(c_{j}-c_{i}\right)=0
$$


where $c_{j}$ is average composition of particle sections in $j$ th bin and $c_{i}$ is average composition of particles in $i$ th bin.

In addition there is also the natural constraint

$$
\sum_{i} p_{i, j}=1
$$

Thus the probability entropy (based on an initially uniform prior with respect to $i$ and $j$ ) is

$$
H=-\sum_{i, j} l_{a, j} p_{i, j} \ln p_{i, j} .
$$

Equation (2) is then

$$
\sum_{i, j} l_{a, j} p_{i, j} c_{i}^{2}=\overline{c^{2}}
$$

Maximising the entropy with respect to the constraints (11), (12) and (14) gives

$$
p_{i, j}=A_{j} \exp \left[\mu_{i}\left(c_{i}-c_{j}\right)+\lambda\left(c_{i}^{2}-\overline{c^{2}}\right)\right] .
$$

The various parameters are all adjusted to ensure that the constraints (11), (12) and (14) are satisfied.

\subsection{Simple model using both linear intercepts and planar sections}

In the previous section particle sections were used as a prior. As linear intercepts provide a worse estimate of the particle composition distribution than the particle sections, a method based only on linear intercepts would expect to behave worse than that based on planar intercepts. However there is an interesting variation that allows us to use both distributions (linear intercepts and planar sections) as priors. The basic idea is to estimate particle composition distribution as a function of variance. The formulation is constructed 
such that when the variance is that for linear intercepts, the composition distribution is the same as that for linear intercepts, and when the variance is that for planar intercepts, the composition distribution is the same as that for planar intercepts. Using our original notation, write this equation as

$$
l_{v, i}=l_{a, i}+\omega l_{a, i}-l_{l, i},
$$

where

$$
\omega=\frac{\operatorname{Var}\left(c_{a}\right)-\operatorname{Var}\left(c_{v}\right)}{\operatorname{Var}\left(c_{l}\right)-\operatorname{Var}\left(c_{a}\right)} .
$$

Note that, for single-sized particles, because

$$
\operatorname{Var}\left(c_{l}\right)>\operatorname{Var}\left(c_{a}\right)>\operatorname{Var}\left(c_{v}\right),
$$

$\omega$ is greater than 0 .

\section{Discussion}

Figure 4 shows the various composition distributions (in cumulative form). The distributions are for linear intercepts, planar sections, actual particles, simple entropy model, combined model (using both planar sections and linear intercepts) and using planar intercepts only. The results show that (for the single case given): the linear intercept composition distribution provides a dreadful estimate of particle composition distribution; the particle section composition distribution, although better, still provides a poor estimate of particle composition distribution; the simple entropy model, although providing an even better estimate of particle composition distribution, is still not adequate; the advanced entropy model for particle sections provides quite a good estimate of particle composition distribution; however, the best estimate is obtained by the entropy method using both particle section composition distribution and linear intercept composition distribution. 


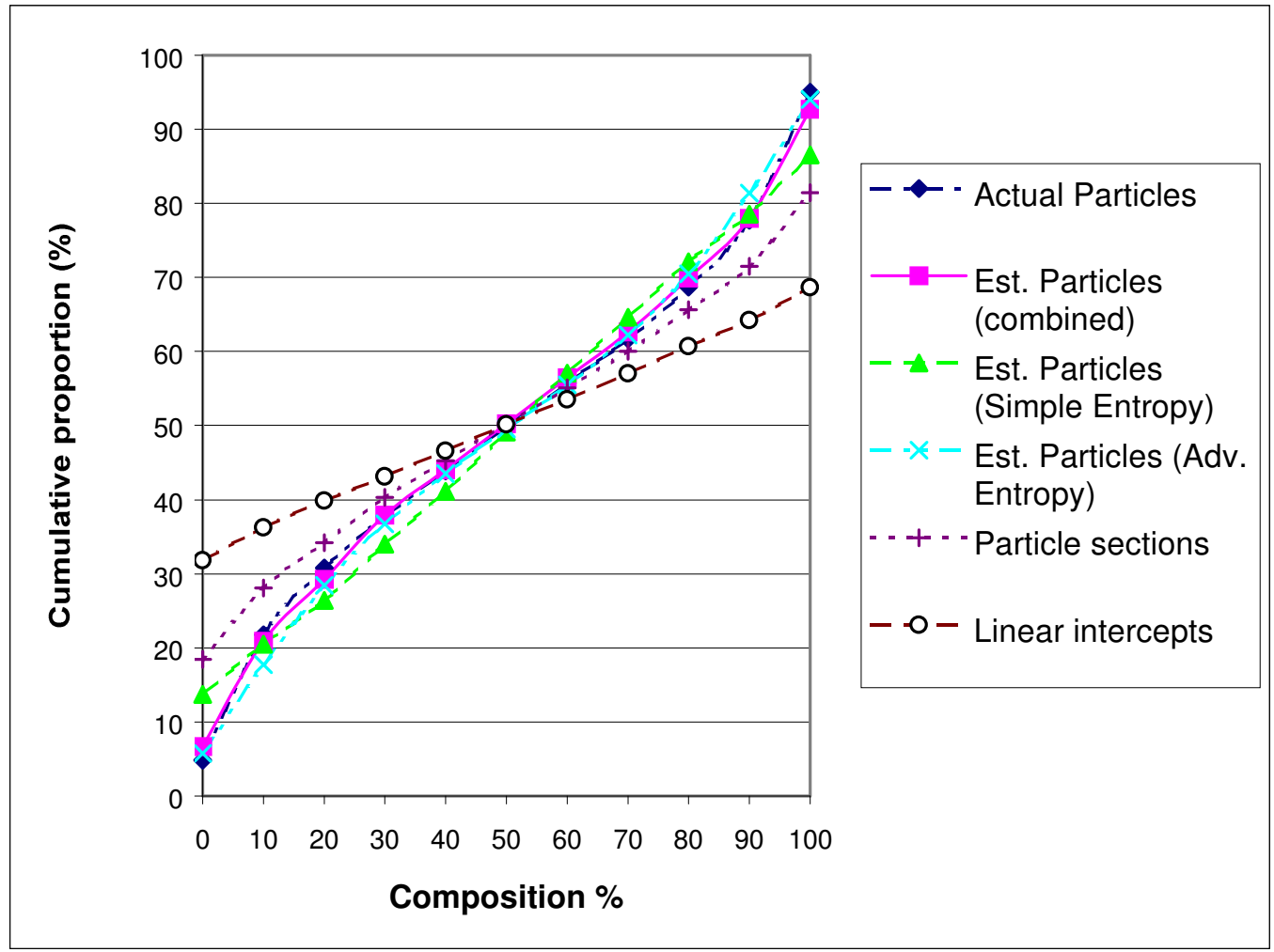

Figure 4: Collated composition distributions for the single test case with simulated spherical caps 


\section{Conclusions}

The results presented here were for a single case, and clearly more comparisons are required before any strong conclusions can be made. However, both the combined model and the advanced entropy model show promise of providing reasonably straightforward methods of stereological correction.

\section{References}

[1] G. Barbery. Mineral Liberation, Les Editions GB, Quebec, 1991.

[2] P. Davy. Probability methods for liberation J. Appl. Prob. 21, pp. 290-296, 1994 C621, C622

[3] M. A. Delesse. Procede machanique pour determiner la composition des roches. C. R. Acad. Sci. 25, p. 544, 1847. C622

[4] A. M. Gaudin Principles of mineral Dressing. MacGraw-Hill, New York. 1939. C621

[5] S. L. Gay. Stereological error in particle sections - the solution. APCOM XXV, pages 313-318, 1995a. C621

[6] S. L. Gay. Stereological equations for phases within particles. J. Microsc. 179: 297-305, 1995b. C623

[7] G. S. Hill, N. Rowland and J. A. Finch. Data correction in two-dimensional liberation studies. Process Mineralogy VII, 617-632, 1990. C621

[8] E. T. Jaynes. Prior Probabilities. IEEE Trans. Syst. Sci. Cybern. 4, 227-241. 1968. C625 
[9] E. T. Jaynes. Probability theory: The logic of science. Available only at website: [Online] http://omega.albany.edu:8008/JaynesBook.html. 1994. C625

[10] M. P. Jones, R. Horton. Recent developments in the stereological assessment of composite (middling) particles by linear measurements. Proc. 11th Cmwlth. Min. and Met. Cong. pp. 113-122, 1979. C621

[11] J. M. Keith. A stereological correction for multiphase particles. PhD thesis, The University of Queensland, 2001. C621, C626

[12] R. P. King and C. L. Schneider. Stereological correction of linear grade distributions for mineral liberation. Powder Technol. 98, 21-37, 1998. C621

[13] J. D.Miller and C. L.Lin Treatment of polished section data for detailed liberation analysis. Int. J. Min. Process. 22: 41-58, 1988. C621

[14] W. Weaver, C. E. Shannon The mathematical theory of communication, University of Illinois Press, Urbana, 1949. C625 